\title{
Some Characterization of the Function Space Type of Lebesgue-Morrey
}

\author{
Rena Eldar Kizi Kerbalayeva ${ }^{1,2}$ \\ ${ }^{1}$ Institute of Mathematics and Mechanics, National Academy Science of Azerbaijan, Baku, Azerbaijan \\ ${ }^{2}$ School №227, Baku, Azerbaijan
}

\section{Email address:}

rena-kerbalayeva@mail.ru

\section{To cite this article:}

Rena Eldar Kizi Kerbalayeva. Some Characterization of the Function Space Type of Lebesgue-Morrey. American Journal of Information Science and Technology. Vol. 5, No. 2, 2021, pp. 25-29. doi: 10.11648/j.ajist.20210502.12

Received: March 11, 2021; Accepted: March 30, 2021; Published: May 14, 2021

\begin{abstract}
As in many areas of Mathematics, we need precise definition and some characterization of function space in order to be absolutely clear. This paper seeks to do that and introduces new definitions and notations to aid our study. I also look at reversible processes of proofs and a new type of function spaces. In this scientific paper we have studied new sub of functions, sub of space of these functions. The new function spaces were investigated in this paper. In the present scientific work we have studied the differentiability functions, some differentiability properties of this type function and in particular, characterization of the differentiability spaces for the functions many groups of variables. At the start of this paper, you will see list of markings that are covered in the paper. Then I gave definition of new normed funksion spase. Following you can see several caharacterization of these spaces and proofs of these caracterization too. Finally, there is connection, known as the Fundamental Analysis, between functions and derivatives of these functions which makes the characterization of functions spaces as a practical tool for science and engineering. The function space is also use to solve many interesting problems some scientific branches like economics, finance, informatics and probability.
\end{abstract}

Keywords: The Space Type of Lebesgue-Morrey, the Function Space of Differentiability Function, Some Properties of These spaces

\section{Introduction}

Let $G \subset R^{n}$ and $1 \leq s \leq n ; s, n$ be naturals, where $n_{1}+\cdots+n_{s}=n$. We consider the sufficient smooth function $\mathrm{f}(\mathrm{x})$, where the point $x=\left(x_{1}, \ldots, x_{s}\right) \in R^{n}$ has coordinates $x_{k}=\left(x_{k .1} ; \ldots ; x_{k, n_{k}}\right) \in R^{n_{k}} \quad\left(\mathrm{k} \in e_{s}=\{1, \ldots, \mathrm{s}\}\right)$. More precisely, $R^{n}=R^{n_{1}} \times R^{n_{2}} \times \cdots \times R^{n_{s}}$. Thus we consider the fixed, non-negative, integral vector $\mathrm{l}=\left(l_{1}, \ldots, l_{s}\right)$ such that, $l_{k}=\left(l_{k .1} ; \ldots ; l_{k, n_{k}}\right), \quad\left(\mathrm{k} \subset e_{s}\right)$ that is, $l_{k, j}>0, \quad(j=$ $\left.1, \ldots, n_{k}\right)$ for all $k \in e_{s}$. Here we consider by $\mathrm{Q}$ the set of vectors $\mathrm{i}=\left(i_{1}, \ldots, i_{s}\right)$ where $\mathrm{i}_{\mathrm{k}}=1,2, \ldots, n_{k}$ for every $k \in e_{s}$. The number of set $\mathrm{Q}$ is equal to: $|Q|=\prod_{k=1}^{S}\left(1+n_{k}\right)$. Therefore, to the vector $\mathrm{i}=\left(i_{1}, \ldots, i_{s}\right) \in Q$, we shall correspond the vector $l^{i}=\left(l_{1}^{i_{1}} ; \ldots ; l_{s}^{i_{s}}\right)$ of the set of non-negative, integral vectors $1=\left(l_{1}, \ldots, l_{s}\right)$, where $l^{0}=$ $(0,0, \ldots, 0), l_{k}^{1}=\left(l_{k, 1}, 0, \ldots, 0\right), \ldots, l_{k}^{i_{k}}=\left(0,0, \ldots, l_{k, n_{k}}\right)$ for all $k \in e_{s}$. Then to the vector $e^{i}$, we let correspond the vector $\bar{l}^{i}=\left(\bar{l}_{1}^{i_{1}}, \bar{l}_{1}^{i_{2}}, \ldots, \bar{l}_{1}^{i_{s}}\right)$, where $\bar{l}_{k}^{i_{k}}=\left(\bar{l}_{k, 1}^{i_{1}}, \bar{l}_{k, 2}^{i_{2}}, \ldots, \bar{l}_{k, n_{k}}^{i_{k}}\right)\left(k \in e_{s}\right)$. Here the largest number $\bar{l}_{k, j}^{i_{k}}$ is less than $l_{k, j}^{i_{k}}$ for all $l_{k, j}^{i_{k}}>0$, when $l_{k, j}^{i_{k}}=0$ then we assume that $\bar{l}_{k, j}^{i_{k}}=0$ for all $k \in e_{s}$. [1,911]

\section{Definition and Preliminaries}

Definition. We denote by

$$
L_{p, a, \varkappa, \tau}(G)
$$

normed Lebesgue-Morrey space of locally summability functions $\mathrm{f}$, on $\mathrm{G}$, with finite norm $\left(N^{i}>l^{i}>m^{i} \geq 0\right.$, $\mathrm{i}=1,2, \ldots, \mathrm{n})$. 


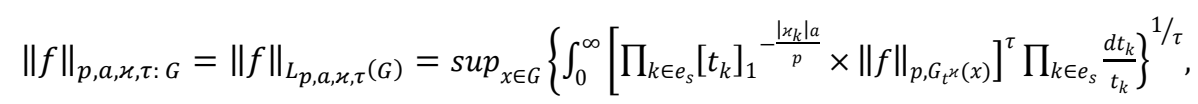

where $\left|\mathcal{H}_{k}\right|=\sum_{j=1}^{n_{k}} \varkappa_{k, j} ;\left[t_{k}\right]_{1}=\min \left\{1, t_{k}\right\} .[2,3,8,12,13]$

\section{Main Results}

Let us give some characterization of $L_{p, a, \varkappa, \tau}(G)$ :

1) For every $\tau>0$ the following inequality take place:

$$
L_{p, a, \varkappa, \tau}(G) \subset_{>} L_{p, a, \varkappa}(G) \subset_{>} L_{p}(G),
$$

that is

$$
\|f\|_{p, G} \leq\|f\|_{p, a, \varkappa: G} \leq C\|f\|_{p, a, \varkappa, \tau: G} .
$$

2) The space $L_{p, a, \varkappa, \tau}(G)$ is complete.

3) For $\mathrm{c}>0$ we have

$$
\|f\|_{p, a, c \mathcal{L}, \tau: G}=\frac{1}{C^{\frac{S}{\tau}}}\|f\|_{p, a, \mathcal{u}, \tau: G} .
$$

4) For any $=\left(\varkappa_{1}, \ldots, \varkappa_{n}\right)>0$ we get:

a) $\|f\|_{p, 0, \mathcal{\varkappa}, \infty: G}=\|f\|_{p, G}$;

b) $\|f\|_{p, 1, \mu_{1} \tau_{-} G} \geq\|f\|_{\infty, G}$.
5) If $p \leq q, \frac{1-a}{q} \leq \frac{1}{p}, 1 \leq \tau_{1} \leq \tau_{2} \leq \infty$ then

$$
L_{q, b, \tau_{,} \tau_{1}}(G) \subset_{>} L_{p, a, \varkappa_{,} \tau_{2}}(G)
$$

and

$$
\|f\|_{p, a, \varkappa, \tau_{2}: G} \leq\|f\|_{q, b, \varkappa, \tau_{1}: G}
$$

Proof 1 We must first proof “(3)”:

$$
\begin{aligned}
& \|f\|_{p, a, \varkappa, \tau: G}^{\tau}=\sup _{x \in G} \int_{0}^{\infty}\left(\prod_{k \in e_{s}}\left[t_{k}\right]_{1}^{-\frac{\left|\varkappa_{k}\right| a}{p}}\|f\|_{p, G_{t} \varkappa(x)}\right)^{\tau} \times \prod_{k \in e_{s}} \frac{d t_{k}}{t_{k}}=\sup _{x \in G}\left\{\int _ { 0 } ^ { \infty } \left(\prod_{k \in e_{s}}\left[t_{k}\right]_{1}{ }^{-\left|\varkappa_{k}\right| a} \times\right.\right. \\
& \left.\left.\int_{G_{t^{\varkappa}}(x)}|f(y)|^{p} d y\right)^{\tau / p}\right\} \prod_{k \in e_{s}} \frac{d t_{k}}{t_{k}} \geq \\
& \sup _{x \in G} \sup _{0<2 t<\infty}\left\{\int_{t}^{2 t} \cdots \int_{t}^{2 t}\left(\prod_{k \in e_{S}}\left[t_{k}\right]_{1}^{-\left|\varkappa_{k}\right| a} \times \int_{G_{t} \varkappa(x)}|f(y)|^{p} d y\right)^{\tau / p}\right\} \prod_{k \in e_{S}} \frac{d t_{k}}{t_{k}} \geq \\
& \left.\prod_{k \in e_{S}} 2^{-\left|\varkappa_{k}\right| a} \sup _{x \in G} \sup _{0<2 t<\infty}\left\{\prod_{k \in e_{S}}\left[t_{k}\right]_{1}^{-\left|\varkappa_{k}\right| a} \times \int_{G_{t} \varkappa(x)}|f(y)|^{p} d y\right)^{\tau / p}\right\} \int_{t}^{2 t} \cdots \int_{t}^{2 t} \prod_{k \in e_{S}}\left[t_{k}\right]_{1}^{-\left|\varkappa_{k}\right| a} \prod_{k \in e_{S}} \frac{d t_{k}}{t_{k}} \geq \\
& \operatorname{Csup}_{x \in G} \sup _{0<2 t<\infty} \prod_{k \in e_{S}}\left(\left[t_{k}\right]_{1}^{-\left|\varkappa_{k}\right| a} \times \int_{G_{t} \mathcal{\varkappa}(x)}|f(y)|^{p} d y\right)^{\tau / p}=C\|f\|_{p, a, \varkappa: G}^{\tau} .
\end{aligned}
$$

Proof 2. Let $\left\{f_{n}\right\}_{n=1}^{\infty}$ be a fundamental consistent in $L_{p, a, \varkappa, \tau}(G)$, that is, for all $\varepsilon>0$, we can take number $n(\varepsilon)$, such that $n, m \geq n(\varepsilon)$,

$$
\sup _{x \in G}\left(\int_{0}^{\infty} \cdots \int_{0}^{\infty} \prod_{k \in e_{S}}\left[t_{k}\right]_{1}^{-\frac{\left|\varkappa_{k}\right| a}{p}} \times\left\|f_{n}-f_{m}\right\|_{p, G_{t} \varkappa(x)}\right)^{\tau} \prod_{k \in e_{S}} \frac{d t_{k}}{t_{k}}<\varepsilon .
$$

Using "(3)" we get 


$$
\left.\sup _{x \in G} \prod_{k \in e_{s}}\left[t_{k}\right]_{1}^{-\frac{\left|x_{k}\right| a}{p}} \times\left\|f_{n}-f_{m}\right\|_{p, G_{t} \varkappa(x)}\right)<\varepsilon
$$

and because of $L_{p, a, \varkappa}(G)$ is complete, we have a function such that $f_{0} \in L_{p, a, \varkappa}(G)$

$$
\prod_{k \in e_{s}}\left[t_{k}\right]_{1}^{-\frac{\left|\varkappa_{k}\right| a}{p}} \times\left\|f_{n}-f_{0}\right\|_{p, G_{t} \varkappa(x)} \rightarrow \rightarrow 0,(n \rightarrow \infty) .
$$

Obviously, for all $t \in(0, \infty)$ and for every $x \in G$, we hold

$$
\left(\prod_{k \in e_{s}}\left[t_{k}\right]_{1}^{-\frac{\left|\varkappa_{k}\right| a}{p}}\left\|f_{n}-f_{0}\right\|_{p, G_{t} \varkappa(x)}\right)^{\tau} \times \prod_{k \in e_{s}} \frac{1}{t_{k}} \rightarrow 0(n \rightarrow \infty) .
$$

Then using theorem Fatou we get

$$
\int_{0}^{\infty} \cdots \int_{0}^{\infty}\left(\prod_{k \in e_{s}}\left[t_{k}\right]_{1}^{-\frac{\left|\varkappa_{k}\right| a}{p}}\left\|f_{n}-f_{0}\right\|_{p, G_{t} \varkappa(x)}\right)^{\tau} \times \prod_{k \in e_{s}} \frac{d t_{k}}{t_{k}} \leq \sup _{m \geq n(\varepsilon)} \int_{0}^{\infty} \cdots \int_{0}^{\infty}\left(\prod_{k \in e_{s}}\left[t_{k}\right]_{1}^{-\frac{\left|\varkappa_{k}\right| a}{p}} \times\left\|f_{n}-f_{m}\right\|_{p, G_{t} \varkappa(x)}\right)^{\tau} \prod_{k \in e_{s}} \frac{d t_{k}}{t_{k}},
$$

for $n \geq n(\varepsilon)$ and for all $x \in G$. Then we have

$$
\sup _{x \in G} \int_{0}^{\infty} \cdots \int_{0}^{\infty}\left(\prod_{k \in e_{S}}\left[t_{k}\right]_{1}^{-\frac{\left|\varkappa_{k}\right| a}{p}}\left\|f_{n}-f_{0}\right\|_{p, G_{t} \varkappa(x)}\right)^{\tau} \times \prod_{k \in e_{S}} \frac{d t_{k}}{t_{k}}<\varepsilon .
$$

Because of $\left\{f_{n}\right\}_{n=1}^{\infty}$ is fundamental consistent in $L_{p, a, \varkappa, \tau}(G)$, then

$$
\left\|f_{0}\right\|_{p, a, \mathcal{\varkappa}, \tau: G} \leq\left\|f_{n}-f_{0}\right\|_{p, a, \mathcal{\varkappa}, \tau: G}+\left\|f_{n}\right\|_{p, a, \mathcal{\psi}, \tau: G} \leq \varepsilon+\mathrm{M},(\mathrm{M}>0),
$$

that is $f_{0} \in L_{p, a, \varkappa, \tau}(G)$.

But it means that, the space $L_{p, a, \varkappa, \tau}(G)$ is complete.

Proof 3.

$$
\begin{aligned}
& \|f\|_{p, a, c \tau, \varkappa: G}=\sup _{x \in G}\left[\left(\int_{0}^{\infty} \ldots \int_{0}^{\infty} \prod_{k \in e_{S}}\left[t_{k}\right]_{1}^{-\frac{c\left|\varkappa_{k}\right| a}{p}}\|f\|_{p, G_{t} \mathcal{H}(x)}\right)^{\tau} \times \prod_{k \in e_{S}} \frac{d t_{k}}{t_{k}}\right]^{1 / \tau}=\sup _{x \in G}\left[\int _ { 0 } ^ { \infty } \ldots \int _ { 0 } ^ { \infty } \left(\prod_{k \in e_{s}}\left[t_{k}^{c}\right]_{1}^{-\frac{\left|\varkappa_{k}\right| a}{p} \times}\right.\right. \\
& \left.\left.\|f\|_{p, G_{t} \mathcal{H}(x)}\right)^{\tau} \prod_{k \in e_{s}} \frac{d t_{k}}{t_{k}}\right]^{1 / \tau}=\sup _{x \in G}\left(\frac{1}{c^{s}} \int_{0}^{\infty} \cdots \int_{0}^{\infty}\left(\prod_{k \in e_{s}}\left[u_{k}\right]_{1}^{-\frac{\left|\mathcal{x}_{k}\right| a}{p}}\|f\|_{p, G_{u} \varkappa(x)}\right)^{\tau} \times\right. \\
& \left.\prod_{k \in e_{s}} \frac{d u_{k}}{u_{k}}\right)^{1 / \tau}=\frac{1}{c^{s / \tau}} \times \sup _{x \in G}\left(\int_{0}^{\infty} \ldots \int_{0}^{\infty}\left(\prod_{k \in e_{s}}\left[u_{k}\right]_{1}^{-\frac{\left|x_{k}\right| a}{p}}\|f\|_{p, G_{u} \varkappa(x)}\right)^{\tau} \times \prod_{k \in e_{s}} \frac{d u_{k}}{u_{k}}\right)^{1 / \tau}=\frac{1}{c^{s / \tau}}\|f\|_{p, a, \varkappa, \tau: G} .
\end{aligned}
$$

Proof 4. It is easy to verify 4(a). Let us proof 4(b). It is known that

$$
\|f\|_{\infty, G} \leq\|f\|_{p, 1, \varkappa: G} .
$$

Then taking the inequality "(3)" then it completes proof 4(b).

Proof 5. We knew that

$$
\|f\|_{p, a, \varkappa: G} \leq\|f\|_{q, b, \varkappa: G},
$$

following for any $\mathrm{t}, 0<t \leq 1$,

$$
\prod_{k \in e_{S}}\left[t_{k}\right]_{1}^{-\frac{\left|\varkappa_{k}\right| a}{p}}\|f\|_{p, G_{t^{\varkappa}}(x)} \leq
$$




$$
\leq \prod_{k \in e_{s}}\left[t_{k}\right]_{1}^{-\frac{\left|\varkappa_{k}\right| b}{q}}\|f\|_{q, G_{t} \varkappa(x)}
$$

then we get

$$
\begin{aligned}
& \prod_{k \in e_{s}}\left[t_{k}\right]_{1}-\frac{\left|\varkappa_{k}\right| a}{p}-\frac{1}{\tau_{1}}\|f\|_{p, G_{t^{\varkappa}}(x)} \leq \\
& \leq \prod_{k \in e_{S}}\left[t_{k}\right]_{1}^{-\frac{\left|\varkappa_{k}\right| b}{q}-\frac{1}{\tau_{1}}}\|f\|_{q, G_{t^{\varkappa}}(x)}, \\
& \sup _{x \in G}\left[\int_{0}^{1} \ldots \int_{0}^{1}\left(\prod_{k \in e_{S}}\left[t_{k}\right]_{1}^{-\frac{\left|\varkappa_{k}\right| a}{p}}\|f\|_{p, G_{t^{\varkappa}}(x)}\right)^{\tau_{1}} \times\right. \\
& \left.\prod_{k \in e_{S}} \frac{d t_{k}}{t_{k}}\right]^{1 / \tau_{1}} \leq \sup _{x \in G}\left[\int _ { 0 } ^ { 1 } \ldots \int _ { 0 } ^ { 1 } \left(\prod_{k \in e_{S}}\left[t_{k}\right]_{1}^{-\frac{\left|x_{k}\right| b}{q} \times}\right.\right. \\
& \left.\left.\|f\|_{q, G_{t} \varkappa(x)}\right)^{\tau_{1}} \prod_{k \in e_{s}} \frac{d t_{k}}{t_{k}}\right]^{1 / \tau_{1}} .
\end{aligned}
$$

Taking

$$
1 \leq \tau_{1} \leq \tau_{2} \leq \infty
$$

then we get "(4)". In addition for every $t>1$

$$
\prod_{k \in e_{S}}\left[t_{k}\right]_{1}^{-\frac{1}{\tau_{1}}}\|f\|_{p, G^{\varkappa}(x)} \leq \prod_{k \in e_{S}}\left[t_{k}\right]_{1}^{-\frac{1}{\tau_{1}}}\|f\|_{q, G_{t} \varkappa(x)}
$$

and

$$
\begin{aligned}
& \sup _{x \in G}\left[\int_{1}^{\infty} \cdots \int_{1}^{\infty}\left(\|f\|_{p, G_{t} \varkappa(x)}\right)^{\tau_{1}} \prod_{k \in e_{s}} \frac{d t_{k}}{t_{k}}\right]^{1 / \tau_{1}} \leq \\
& \leq \sup _{x \in G}\left[\int_{1}^{\infty} \cdots \int_{1}^{\infty}\left(\|f\|_{q, G} t^{\varkappa(x)}\right)^{\tau_{1}} \prod_{k \in e_{s}} \frac{d t_{k}}{t_{k}}\right]^{1 / \tau_{1}} .
\end{aligned}
$$

Here again using $1 \leq \tau_{1} \leq \tau_{2} \leq \infty$ then we get "(4)". [4, 5, $6,8,14,15]$

\section{Conclusion}

The properties given normed Lebesgue-Morrey space allow us to introduce some new normed spaces. In addition, we can give some properties of these type spaces and prove these properties. We can carry out the same procedures in the prove of the characterization of some spaces to show the inequality "3" and Fatou theorem. All of these concepts, of course, are underpinned by the connected properties of a function with many group variables. It is the author's options that the concepts of given normed Lebesgue-Morrey space provide a vehicle for generalizing Lebesgue space to the normed spaces setting and ultimately suggest generalizations to the most important case of functions with many group variables on Lebesgue spaces. Present work by the author is devoted to generalizing to the concepts of Lebesgue spaces with many group variables to introduce if more refined results on the support and the property of this type space can be obtained.

\section{Acknowledgements}

The author wishes to thank Professors A. Dj. Djabrailov, A. M. Najafov for several conversations, their hospitality and generous support.

\section{References}

[1] Djabrailov, A. Dj. (1993) The method of integral representation in the theory of spaces of function of several groups variables. Kluwer Academic Publishers, $13-79$.

[2] Fazio, G Di., Ragua, M. (1993) Interior estimates in Morrey spaces for strong solutions to nondevergence form equation with discontinuous coefficients. Journal Func. Anal., 11, 105115 . 
[3] Fan Di., Lu S., Yang D. (1998) Regularity in Morrey spaces of strong solutions to nondivergence elliptic equations with VMO coefficients. Georgian Math. Jour., 5, 425-440.

[4] Guiseppe, S. (1998) Un problema di Darboux in un insieme non limitato. Esistenza, unicita e dipendenza continua della soluzione. Math., 53 (2), 359-373.

[5] Kato T. (1992) Strong solutions of the Navier-Stokes equation in Morrey. Soc. Brasil. Mat., 22, 127-155.

[6] Kozano H. (1994) Comm. Partial Differential Navier-Stokes equations. Yamasaki M. Semilinear heat equations and the Navier-Stokes equation with distributions in new function spaces as initial data, 19, 959-1014.

[7] Lin Tang., Jingshi Xu. (2005) Some properties of Morrey type Besov-Triebel spaces. Math. Nachr., 278 (7/8), 904-917.

[8] Mamedov I. G. (2002) The local boundary value problem for an integro-differential equation. Proceedings of Inst. of mathematics and mechanics, XVIII, 96-101.

[9] Mazzucato, A. I. (2001) Decomposition of Besov-Morrey spaces. Proceedings of Conference on Harmonic Analysis, 215-233.
[10] Najafov, A. M. (2005) On some properties of the function from Sobolev-Morrey type spaces. Central Europen Journal of Mathem., 3 (3), 496-507.

[11] Najafov A. M. (2005) Some properties of functions from the intersection of Besov-Morrey type spaces with dominant mixed derivatives. Proceedings of A. Razmadze Math. Inst., $139,71-82$.

[12] Najafov A. M. (2005) Problem on smoothness of solution of one class hypoelliptic equations. Proceedings of A. Razmadze Math. Inst., 140, 131-139.

[13] Najafov A. M., Kerbalayeva R. E. (2015) Interpolation theorems for spaces Besov-Morrey type. Journal presented by institute Mathematics and Computer Sciences at TskhumAbkhazian Academy of Sciences, Tskhum-Abkhazian, IX-X, 198-212.

[14] Najafov A. M., Kerbalayeva R. E. (2019) The embedding theorems for Besov-Morrey spaces of many groups of variables. Proceedings of A. Razmadze Institute Mathematics. Georgian Academy of Sciences, 26(1), 125-131.

[15] Taylor, M. (1994/1995) Microlocal analysis on Morrey spaces. Singularities and oscullations (Minneapolis). IMA 91, Math. Appl., Springer, New Yor., 97-135. 